\title{
Wavelet Transforms and Signal Ambiguity Functions
}

\author{
Arjuna Peter Chaiyasena, Leon H. Sibul, Augustine Banyaga ${ }^{1}$, and Mark L. Fowler
}

\author{
Applied Research Laboratory and \\ The Department of Mathematics ${ }^{1}$ \\ The Pennsylvania State University \\ P.O. Box 30 \\ State College, PA 16804
}

\begin{abstract}
Classical results from group representation theory are used to gain insight into important properties of narrowband and wideband ambiguity functions and wavelet transforms. Wideband ambiguity functions are essentially affine wavelet transforms and narrowband ambiguity functions can be considered to be Heisenberg wavelet transforms. Important invariance properties of the ambiguity functions are consequences of the group representation theory. This paper presents examples of derivations of wideband ambiguity function properties.
\end{abstract}

\section{INTRODUCTION}

There has been a recent explosion of research interest in continuous and discrete wavelet transforms $[1,2,3,4,5,6,7]$. The wavelet transforms can be considered to be time-frequency localization operators that treat the time-frequency plane (phase space or in terms of Gabor, the information plane) as one geometric whole rather than as two scparate spaces [5]. Localization of signals in the time $x$ frequency plane is the essence of the signal ambiguity function problem. In the case of narrowband ambiguity functions, one computes the inner product of a received signal with a time delayed and Doppler shifted version of the reference signal $g(t)$.

All the time delayed and Doppler shifted signals can be obtained from the elementary signal $g(t)$ by a unitary operator that represents a Heisenberg group clement. Thus, the narrowband ambiguity function can be interpreted as the generalized Gabor transform of the received signal with respect to the reference signal $g(t)$.

Gabor originally used $g(t)=e^{-r t^{2}}$ because it has the minimum possible product of rms duration and bandwidth [9]. For this reason also, Gabor transforms have fundamental significance in physics and information theory. Later, Montgomery and Reed [10] showed that the Gabor transform is well defined for any reference signal $g(t) \in L^{2}(\mathcal{R})$; this result is easily verified using the theory of group representations [1]. To emphasize the connection to the Heisenberg group, we will refer to the Gabor transform as the Heisenberg wavelet transform.

In the case of wideband ambiguity functions, one computes the inner product of a received signal with a time delayed and dilated version of the reference signal $g(t)$. These time delayed and dilated signais can be obtained from $g(t)$ by a unitary operator that represents an affine group element. Hence wideband cross-ambiguity functions are affine wavelet transforms. Auto-ambiguity functions are special cases of cross-ambiguity functions.

Group representation theory provides a unified framework for the study of narrowband and wideband ambiguity functions, as well as Heisenberg and affine wavelet transforms. The important concept is that both narrowband and wideband ambiguity functions are coefficients of the unitary representations of their respective groups. Various well known invariance properties of narrowband and wideband ambiguity functions follow easily from this fact. We demonstrate the latter by several examples.

Group representation theory also helps to explain the essential differences between narrowband and wideband ambiguity functions and related wavelet transforms. In particular it gives us new insight into the ambiguity volume conservation and admissible signals. Ambiguity volume conservation is a property of practical significance for the synthesis of realizable signals with desired ambiguity properties.

The objective of this article, an up-dated version of our earlier paper [21], is to provide a unified group theoretic treatment of ambiguity functions and wavelet theory. Additional results that are presented here are group theoretic derivations of ambiguity function invariance properties.

The work makes use of the results of considerable recent literature on this topic $[1,2,3,4,5,6,7]$. Heil and Walnut [1], for instance, present a good up-to-date overview of wavelet transform research in a paper highly recommended to readers interested in this fascinating topic. Group theoretic aspects of narrowband ambiguity functions have been investigated also by Auslander and Tolimieri [11] and by Schempp [12, 13, 14]. Our discussion emphasizes the connection between affine wavelet transforms and wideband ambiguity functions, with the key integral formulas to be discussed in this paper being:

Narrowband Ambiguity Function:

$$
H(f, g ; x, y)=\int_{-\infty}^{\infty} f(t)[g(t-x)]^{*} e^{-2 \pi i y t} d t
$$

Wideband Ambiguity Function:

$$
W(f, g ; s, \tau)=s^{-1 / 2} \int_{-\infty}^{\infty} f(t)[g(s t+\tau)]^{*} d t
$$




\section{SOME MATHEMATICAL BACKGROUND}

Basic knowledge of group theory as presented in [15], and knowledge of Hilbert space in [16] and [17] will be assumed. However, the definition of a group will be reviewed.

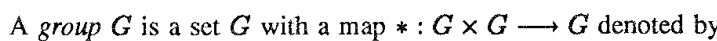
$(x, y) \longmapsto x * y$ having the following properties:

1. Closure: $x * y \in G$ for all $x, y \in G$.

2. Associativity: $(x * y) * z=x *(y * z)$.

3. There is an element $e \in G$, to be called the identity, such that $e * x=x * e=x$ for all $x \in G$.

4. For each $x \in G$, there is an element, $x^{-1}$, to be called the inverse of $x$ such that: $x * x^{-1}=x^{-1} * x=e$.

If in addition, $x * y=y * x$ for all $x, y \in G$, the group is said to be commutative.

When the operation * is totally clear, the symbol $*$ will be omitted: $x y$ instead of $x * y$.

A subset $S$ of group $G$ is a subgroup of $G$ if it is a group under the same operation as $G$.

The group $G$ may have additional structure. For instance, if $G$ is a topological space and * is continuous with respect to this topology, $G$ will be a topological group. Furthermore, if $G$ has a differentiable structure, $G$ will be a differentiable manifold, and called a Lie Group.

A differentiable manifold for our purpose is a set which "looks like" Euclidean space. For example, $\mathcal{R}^{n}$ is one since it is Euclidean space. On Euclidean space, we can choose different parametrizations; however, the change of coördinates must be differentiable. Rectangular coördinates and polar coördinates are familiar examples of such parametrizations of $\mathcal{R}^{2}$. We recall that change of coördinates must also be invertibl e locally, that is, that the Jacobian of the transformation be invertible.

There is a vast amount of theory of Lie groups. For purposes of this paper, we will concentrate on a special category of groups called the linear groups. These are groups which can be realized as groups of linear transformations of a vector space, or equivalently, as matrix groups.

The set $G l(n, \mathcal{R})$ of invertible matrices with real coefficients form a group called the real general linear group, under the usual matrix multiplication. Indeed, the set $G l(n, \mathcal{R})$ may be parametrized using $n^{2}$ coordinate functions, by fixing a basis in $\mathcal{R}^{n}$. Thus $\operatorname{Gl}(n, \mathcal{R})$ "looks like" $\mathcal{R}^{n^{2}}$.

It is a fact that every group of invertible linear transformations of a real n-dimensional vector space may be seen as a subgroup of $G l(n, \mathcal{R})$.

The subgroup of $\operatorname{Gl}(2, \mathcal{R})$ consisting of matrices of the form

$$
\left(\begin{array}{ll}
s & t \\
0 & 1
\end{array}\right)
$$

with $s>0$ and $t$ any real number, is called the real affine group. It will be denoted by $\mathcal{A}^{1}$. Its identity clement is the usual identity element of $G l(2, \mathcal{R})$, and the inverse of an element is

$$
\left(\begin{array}{cc}
s^{-1} & -s^{-1} t \\
0 & 1
\end{array}\right)
$$

Another matrix group of particular interest to this paper is the so-called Heisenberg group, $\mathcal{H}$, the group of matrices of the form

$$
\left(\begin{array}{lll}
1 & x & z \\
0 & 1 & y \\
0 & 0 & 1
\end{array}\right)
$$

It can be verified that the identity and inverse of this set belongs to $\mathcal{H}$, and that a parametrization of the Heisenberg group is the one that associates a matrix with its three superdiagonal entries. One sees, then, that $\mathcal{H}$ "looks like" $\mathcal{R}^{3}$.

The set of operators on a Hilbert space that preserve its inner product forms a group under composition, called the Unitary Group of the Hilbert space involved. Specifically, $L^{2}(\mathcal{R})$ will be our Hilbert space under consideration.

It is possible to define measures on topological groups and hence Lie groups, such as the Heisenberg group and the affine group. These measures are compatible with the group structure in the sense that they remain unchanged if the set is translated by a multiplication by a group element. Since groups are not commutative in general, there are both left invariant and right invariant measures. If $f$ is a measurable function on a group $G, \mu$ is left-invariant, if

$$
\int_{G} f\left(g_{0} * g\right) d \mu(g)=\int_{G} f(g) d \mu(g)
$$

for all $g_{0} \in G$. The right invariant measure is defined similarly. If an invariant measure, left or right, exists for a group, it is well known that it must necessarily be unique up to multiplication by a constant. Finally, if the left and right invariant measure coincide, the group in question is said to be unimodular.

An example of left invariant measure is the well known Lebesgue (or Riemann) measure. The group involved is the group of real numbers under addition. Certainly,

$$
\int_{-\infty}^{\infty} f(x+y) d x=\int_{-\infty}^{\infty} f(x) d x
$$

for all $y \in \mathcal{R}$. This group is unimodular since the real numbers are commutative under addition.

A unitary representation $U$ of a group $G$ on a Hilbert space $H$ is a mapp ing assigning to each group element a unitary operator $U(x)$ on $H$, such that for any $x, y \in G$,

$$
U(x * y)=U(x) \circ U(y) .
$$

(Recall that $U$ is a unitary operator on $H$ if, for all $v, w \in H,\langle U v, U w\rangle$ $=\langle v, w\rangle)$.

A subspace $S$ of $H$ is invariant under $U$ if for any $v \in S, U(x)$. $v \in S$ for all $x \in G$. A representation $U$ is said to be irreducible if the only invariant subspaces of $H$ are the zero subspace and $H$ itself.

An irreducible unitary representation $U$ of a group $G$ on a Hibert space $H$ is said to be square integrable if there is some $g \in H$ such that: 


$$
\int_{G}|\langle g, U(x) g\rangle|^{2} d \mu(x)<\infty,
$$

where $\mu$ is the left invariant measure of $G$. Such a $g$ is said to be an admissible vector.

Square integrable representations enjoy the following property, described by a generalization of the Frobenius-Schur-Godement theorem [13]: If $U$ is a square integrable representation of a group $G$ acting on a Hilbert space $H$, there exists a unique self-adjoint positive operator $Q$ such that:

i) The set of admissible vectors coincide with the domain of $Q$.

ii) Let $g_{1}$ and $g_{2}$ be two admissible vectors. Let $f_{1}$ and $f_{2}$ be any vectors in $H$, then

$$
\int_{G}\left\langle f_{1}, U(x) g_{1}\right\rangle\left\langle f_{2}, U(x) g_{2}\right\rangle^{*} d x=\left\langle Q g_{2}, Q g_{1}\right\rangle\left\langle f_{1}, f_{2}\right\rangle .
$$

Finally, if $G$ is unimodular, then $Q$ is a multiple of the identity operator, hence the domain of $Q$ will be all of $H$, that is, all of $H$ constitutes the set of admissible vectors for the representation.

The above theorem is proved in detail in [2]. It will play a major role in determining the volume under the ambiguity surface.

The coefficient of a continuous unitary group representation $U$ of $G$ on a Hilbert space $H$ with respect to the ordered pair $(f, g)$, both $f$ and $g$ in $H$, is a mapping $C_{U, f, g}: G \longrightarrow \mathcal{R}$ such that

$$
x \mapsto\langle f, U(x) g\rangle .
$$

This coefficient is a generalization of the Fourier coefficients, and will be useful in rephrasing well known concepts later.

For notational convenience, we define the following operators on $L^{2}(\mathcal{R})$ :

- Translation: $T_{a} f(x)=f(x-a), a \in \mathcal{R}$.

- Dilation: $D_{k} f(x)=\sqrt{k} f(k x), k>0$.

- Modulation: $E_{\alpha} f(x)=e^{2 \pi i a x} f(x), a \in \mathbb{R}$.

- Muliplication: $M_{a} f(x)=e^{2 \pi i a} f(x), a \in \mathcal{R}$.

These operators are easily seen to be unitary.

Further information concerning group representations may be found in [18].

\section{THE WIDE AND NARROW BAND AMBIGUITY FUNCTIONS}

It is the purpose of this section to show that the wide and narrow band ambiguity functions are the cocfficients of the affine and Hcisenberg group respectively. This essentially is the vital connection between the functions and group theory.

Consider the Heisenberg group, $\mathcal{H}$. It has a unitary representation $U$ on $L^{2}(\mathcal{R})$ as follows: $U(x, y, z)$ is the unitary operator corresponding to $(x, y, z)$ in $\mathcal{H}$ such that:

$$
\begin{aligned}
U(x, y, z) f(t) & =M_{x} T_{x} E_{y} f(t) \\
& =z e^{2 \pi i y(t-x)} f(t-x) .
\end{aligned}
$$

That this unitary representation of $\mathcal{H}$ is unique in a certain sense and irreducible is the content of the Stone-Von Neuman Theorem [7]

When we apply the definition of coefficient to $U$ on $L^{2}(\mathcal{R})$ with respect to $(f, g)$, we obtain

$$
\begin{aligned}
C_{U, f, g} & =\langle f, U(x, y, z) g\rangle \\
& =\int_{-\infty}^{\infty} f(t)\left[M_{z} T_{x} E_{y} g(t)\right] * d t \\
& =\int_{-\infty}^{\infty} f(t)[z g(t-x)]^{*} e^{-2 \pi i y(t-x)} d t .
\end{aligned}
$$

When we set $z=1$, we find that the coefficient takes the form of the narrow band cross ambiguity function. Of course, letting $f=g$ leads to the narrow band ambiguity function, a connection with the Heisenberg group which allows us to discover many properties.

One of the more important of these properties is the volume conservation property of the narrow band ambiguity functions. To derive this abstractly, one must prove that the representation $U$ of $\mathcal{H}$ is square integrable. However, since the parametrization in the previous section does not lead to a square integrable representation, it is necessary to display a new parametrization as follows:

$$
(x, y, z) \mapsto\left(x, y, e^{2 \pi i z}\right) .
$$

This parametrization changes the multiplication rule:

$$
\left.\left(x, y, e^{2 \pi i z}\right) *\left(x^{\prime}, y^{\prime}, e^{2 \pi i z^{\prime}}\right)=\left(x+x^{\prime}, y+y^{\prime}, e^{2 \pi i\left(x+x^{\prime}+x y^{\prime}\right.}\right)\right) .
$$

As one can see by the above, the reparametrization really only consists of exponentiating the third component of each element.

Under this reparametrization, the left and right invariant measure of $\mathcal{H}$ can be computed to be: $d x d y d z / 2 \pi$, the proof of which is contained in [1]. This, by the way, shows an example of a unimodular group which is not commutative. By the Frobenius-Schur-Godement theorem, all of $L^{2}(R)$ is the set of admissible vectors. An explicit proof is presented in [1]. $\mathcal{H}$, then

By the same theorem, substituting appropriately for the case of

$$
\begin{gathered}
\int_{0}^{1} \int_{-\infty}^{\infty} \int_{-\infty}^{\infty}\left\langle f_{1}, U(x, y, z) g_{1}\right\rangle\left\langle f_{2}, U(x, y, z) g_{2}\right\rangle^{*} d x d y d z / 2 \pi \\
=\left\langle g_{2}, g_{1}\right\rangle\left\langle f_{1}, f_{2}\right\rangle .
\end{gathered}
$$

If we let $f=f_{i}=g_{i}, i=1,2$; then we obtain:

$$
\int_{0}^{1} \int_{-\infty}^{\infty} \int_{-\infty}^{\infty}|\langle f, U(x, y, z) f\rangle|^{2} d x d y d z / 2 \pi=\|f\|^{4} .
$$
fies to:

If we further assume that $\|f\|=1$, the above expression simpli-

$$
\int_{0}^{1} \int_{-\infty}^{\infty} \int_{-\infty}^{\infty}|\langle f, U(x, y, z) f\rangle|^{2} d x d y d z / 2 \pi=1
$$

This is the statement of the conservation of ambiguity volume. Seen in this context, the conservation property is true of any "ambi- 
guity function" which is a matrix coefficient of a unimodular group.

The conservation of narrowband ambiguity volume having been proved, it is natural to ask whether a similar conservation law holds for wide band ambiguity functions. Unfortunately, the answer is no, based on group theoretic reasoning.

The Affine group, $\mathcal{A}^{1}$ has a unitary representation $A$ on $L^{2}(\mathcal{R})$ as follows: $A(s, \tau)$ is the unitary operator corresponding to $(s, \tau)$ in $\mathcal{A}^{1}$ of the form:

$$
\begin{aligned}
A(s, \tau) f(t) & =T_{\tau} D_{s} f(t) \\
& =\sqrt{s} f(s t+\tau) .
\end{aligned}
$$

As in the case of $\mathcal{H}$, we form the coefficient of this representation:

$$
\begin{aligned}
C_{A, f g} & =\langle f, A(s, \tau) g\rangle \\
& =\int_{-\infty}^{\infty} f(t)\left[T_{\tau} D_{s} g(t)\right]^{*} d t \\
& =\int_{-\infty}^{\infty} f(t)[\sqrt{s} g(s t+\tau)]^{*} d t
\end{aligned}
$$

which is the wavelet transform of $f$ with respect to $g$.

One immediately sees that this coefficient is no other than the wide band ambiguity function.

The group $\mathcal{A}^{1}$ may be reparametrized in the form:

$$
(s, \tau) \mapsto\left(e^{-s}, \tau\right),
$$

where $s$ can take on all real values. The corresponding left invariant measure can be shown to be $e^{s} d s d \tau$ and the right invariant measure is $d s d \tau . \mathcal{A}^{1}$ is thus not a unimodular group. It is proved in [1] that the admissible vectors of the representation above are those $g$ such that:

$$
\int_{0}^{\infty} \frac{\hat{g}(\omega)}{\omega} d \omega<\infty
$$

where $\hat{g}(\omega)$ denotes the Fourier transform of $g(t)$.

Letting $s$ and $\tau$ vary, $C_{A, f, g}$ traces a surface in space called the wideband ambiguity surface. Unlike the narrowband case, the volume under this surface for $\|f\|=1$ is not constant. Indeed the same calculation proving the square integrability of $A$ yields:

$$
\int_{-\infty}^{\infty} \int_{-\infty}^{\infty}|\langle f, A(s, \tau) g\rangle|^{2} d s d \tau=\int_{0}^{\infty}|\hat{f}(\omega)|^{2} d \omega \int_{0}^{\infty} \frac{|\hat{g}(\gamma)|^{2}}{|\gamma|} d \gamma
$$

Letting $f=g$, we obtain the volume formula:

$$
\left.\int_{-\infty}^{\infty} \int_{-\infty}^{\infty}\left|\langle f, A(s, \tau) f\rangle^{2} d s d \tau=\int_{0}^{\infty}\right| \hat{f}(\omega)\right|^{2} d \omega \int_{0}^{\infty} \frac{|\hat{f}(\gamma)|^{2}}{|\gamma|} d \gamma
$$

which agrees with an independent calculation by Sibul and Titlebaum [19]. Hence wideband ambiguity is not conserved.

Thus some properties of ambiguity functions may be traced back to properties of groups they represent.

\section{INVARIANCE PROPERTIES}

In [20], Altes proved various invariance properties of the wideband ambi guity function. These results can also be viewed as a consequence of the wideband ambiguity function bcing the coefficient of a unitary representation of the affine group. However, Altes used the formula:

$$
W_{f, g}=\int_{-\infty}^{\infty} f(t) g^{*}[s(t+\tau)] d t
$$

instead of:

$$
W_{f, g}=\int_{-\infty}^{\infty} f(t) g^{*}(s t+\tau) d t
$$

Both forms are used in the engincering literature. The second form, however, is a coefficient of the affine group, and so exhibits much symmetry. We will focus on the second form, however it is not difficult to transform it to the first by a change of variable.

As a first example, Altes proved that $W_{f, g}(s, \tau)=$ $W_{g, f}^{*}\left(s^{-1},-\tau / s\right)$. This fact can be seen from a group theoretic point of view as follows:

$$
W_{f, g}(s, \tau)=\langle f, A(s, \tau) g\rangle .
$$

But $A$ is a unitary representation of the affine group and so the inverse of $A$ is equal to the adjoint of $A$ and

$$
\begin{aligned}
\langle f, A(s, \tau) g\rangle & =\left\langle[A(s, \tau)]^{-1} f,[A(s, \tau)]^{-1} A(s, \tau) g\right\rangle \\
& =\langle A(1 / s,-\tau / s) f, g\rangle \\
& =\langle g, A(1 / s,-\tau / s) f\rangle^{*} \\
& W_{g, f}^{*}(1 / s,-\tau / s)
\end{aligned}
$$

It can thus be seen that this proof gives some indication of the symmetries underlying the ambiguity function.

The symmetry exploitation idea may be extended by examining relationships between various subgroups of the affine group. The subgroup of dilations, the matrices of the form

$$
\left(\begin{array}{cc}
k & 0 \\
0 & 1
\end{array}\right)
$$

has a unitary representation $D$ on $L^{2}((\mathcal{R}))$ :

$$
D_{k} f(t)=\sqrt{k} f(k t) \text {. }
$$

But it can be shown that $D_{k} \circ A(s, \tau)=A(s, \tau) \circ D_{k}$ by multiplying matrices. Hence the representations of the dilation subgroup and the affine group commute as well. This is the basis for Altes' result: If $f(t)=k^{1 / 2} u(k t)$ and $g(t)=k^{1 / 2} v(k t)$, then

$$
\left|W_{g, f}(s, \tau)\right|^{2}=\left|W_{v, u}(t, s)\right|^{2} .
$$

Indeed,

$$
\begin{aligned}
W_{g, f}(s, \tau) & =\langle g, A(s, \tau) f\rangle \\
& =\left\langle D_{k} v, A(s, \tau) D_{k} u\right\rangle \\
& =\left\langle D_{k} v, D_{k} A(s, \tau) u\right\rangle \\
& =\langle v, A(s, \tau) u\rangle \\
& =W_{v, u}(s, \tau) .
\end{aligned}
$$


Our final example will illustrate another aspect of group theory. A unitary representation of a Lie group $G$ can be seen as a map from $G$ to the unitary group of $L^{2}(\mathcal{R})$ possessing nice properties such as differentiability, and preservation of group structures. Technically, such a map is called a differentiable homomorphism, although such terminology need not concem us here. The image of this type of map is known to be a subgroup of the unitary group, therefore it is possible to investigate relationships between the unitary representation operators and other unitary operators. $L^{2}(R)$ :

One such operator is the "inversion operator" $I: L^{2}(\mathcal{R}) \longrightarrow$

$$
I[f(t)]=f(-t)
$$

$\mathcal{I}$ is not a representation of the affine group since it is equivalent to a negative dilation. However, we can prove that:

$$
A(s, \tau) \circ \mathcal{I}=\mathcal{I} \circ A(s,-\tau)
$$

Indeed,

$$
\begin{aligned}
A(s, \tau) \circ I[f(t)] & =A(s, \tau)[f(-t)] \\
& =f(-s t+\tau) \\
& =f(-(s t-\tau)) \\
& =\mathcal{I} f(s t-\tau) \\
& =\mathcal{I} \circ A(s,-\tau)[f(t)]
\end{aligned}
$$

which was to be proven.

This fact can be used to deduce one of Altes' results: If $f(t)=$ $u(-t)$ and $g(t)=v(-t)$, then $W_{g, f}(s, \tau)=W_{v, u}(s,-\tau)$. The idea of the proof is similar to the others:

$$
\begin{aligned}
W_{g, f}(s, \tau) & =\langle g, A(s, \tau) f\rangle \\
& =\langle\mathcal{I} u, A(s, \tau) \mathcal{I} v\rangle \\
& =\langle\mathcal{I} u, I A(s,-\tau) v\rangle \\
& =\langle u, A(s,-\tau) v\rangle \\
& =W_{u, v}(s,-\tau) .
\end{aligned}
$$

The next to last step follows from the fact that $\mathcal{I}$ is a unitary operator.

Many other properties of the wide band ambiguity function may be proved using these ideas. In fact, similar properties of the narrow band ambiguity function may be obtained by the fact that it is the coeficient of a representation of the Heisenberg group. Invariance properties of the wide and narrow band ambiguity functions may be discovered and proved by investigating properties of the group elements within the groups to which they are related.

\section{CONCLUSIONS}

Widcband and narrowband ambiguity functions are time $\times$ frequency plane localization operators, as are corresponding wavelet transforms. Wideband ambiguity functions are affine wavelet transforms and narrowband ambiguity functions are generalized Gabor transforms or Heisenberg wavelet transforms. In this and in a previous paper [21] we have shown that group representation theory provides a unified framework for the study of all above-mentioned time $x$ frequency plane (phase space) localization operators. In particular, group representation theory has provided new insight into admissible signals, ambiguity volume conservation, and ambiguity function invariance properties.

\section{ACKNOWLEDGEMENTS}

This work has been supported by the Office of Naval Research under the Fundamental Initiatives Program and by NSF grant DMS9001861. We would like to thank Randy Young for his suggestion that we demonstrate for the signal processing community how easy it is to derive invariance properties of signal ambiguity functions through the use of group representation theory.

\section{REFERENCES}

[1] C. E. Heil and D. F. Walnut; "Continuous and discrete wavelet transforms," SIAM Review, vol. 31, No. 4, December 1989 , pp. 628-666.

[2] A. Grossmann, J. Morlet, and T. Paul, "Transforms associated to square integrable group representations, I: General Results," J. Math. Physics, vol. 26, October 1985, pp. 2473-2479.

[3] A. Grossmann, J. Morlet, and T. Paul, "Transforms associated to square integrable group representations, II: Examples," Ann. Inst. Henri Poincare, Vol. 45, No. 3, 1986, pp. 293-309.

[4] A. Grossmann and J. Morlet, "Decomposition of Hardy functions into square integrable wavelets of constant shape," SIAM J. Math Analysis,, Vol. 45, No. 4, July 1984, pp. 723-736.

[5] I. Daubechies, "Time-frequency localization operators: A Geometric phase space approach," IEE Trans. on Information Theory, Vol. 34, No. 4, 1988, pp. 605-612.

[6] I. Daubechies, A. Grossmann, and Y. Meyer, "Painless nonorthogonal expansions," J. Math. Phys., Vol. 27, No. 5, May 1986, pp. $1271-1283$.

[7] G. B. Folland, Harmonic Analysis on Phase Space; Annals of Mathematics Studies, No. 122, Princeton University Press, 1989, Princeton.

[8] D. Gabor, "Theory of communication," IEE (London) 93 (III), November 1946, pp. 429-457.

[9] C. W. Helstrom, "An Expansion of a signal in gaussian elementary signals," IEEE Trans. on Information Theory, Vol. IT-12, January 1966, pp. 81-82.

[10] L. K. Montgomery, Jr. and I. S. Reed, "A Generalization of the Gabor-Helstrom transform," IEEE Trans. on Information Theory, Vol. IT-13, pp. 344-345, April 1967.

[11] L. Auslander and R. Tolimieri, "Radar ambiguity functions and group theory," SIAM J. Math Anal., Vol. 16, No. 3, May 1985, pp. 577-601.

[12] W. Schempp, Harmonic Analysis on the Heisenberg Nilpotent Lie Group, Pitman Research Notes in Mathematics Series, Longman Scientific and Technical, Great Britain, 1986. 
[13] W. Schempp, "Analog radar design and digital signal processing-a Heisenberg nilpotent Lie group approach," In Lie Methods in optics, Chapter 1. J. Sanchez-Mandragon and K.B. Wolf, cds. Lecture Notes in Physics. Springer, Berlin Hcidelberg - New York - Tokyo, 1986.

[14] W. Schempp, "Radar Ambiguity Functions, The Heisenberg Group, and Holomorphic Theta Series," Proc. American Mathematical Society, Vol. 92, No. 1, September 1984, pp. 103-110.

[15] R. Hermann, General Algebraic Ideas, Interdisciplinary Mathematics, Vol. 1, Math Sci. Press, Massachusetts, 1973.

[16] P. R. Halmos. Introduction to Hilbert Space and the Theory of Spectral Multiplicity. Chelsea Publishing Company, New York, 1957.

[17] G. F. Simmons. Introduction to Topology and Modern Analysis, Robert E. Krieger Publishing Company, Florida, 1986.

[18] A. A. Kirillov, Elements of the Theory of Representations, Springer-Verlag: Berlin - Heidelberg - New York, 1976.

[19] L. H. Sibul and E. L. Titlebaum, "Volume properties for the wideband ambiguity function," IEEE Trans, on Aerospace and Electronic Systems, Vol. AES-17, No. 1, January 1981, pp. 83-86.

[20] R. A. Altes, "Some invariance properties of the wide-band ambiguity function," The Journal of the Acoustical Society of America, Vol 53, No. 4, 1973, pp. 1154-1160.

[21] L. H. Sibul, A. P. Chaiyasena, and M. L. Fowler, "Signal ambiguity functions, Wigner transforms, and wavelets," Signal Processing and Digital Filtering, M. H. Hamza, editor. Lugano, Switzerland, June 1990, pp. 214-217. 